\title{
Correction to: Early presence of anti-angiogenesis-related adverse events as a potential biomarker of antitumor efficacy in metastatic gastric cancer patients treated with apatinib: a cohort study
}

Xinyang Liu ${ }^{1,2}$, Shukui Qin ${ }^{3}$, Zhichao Wang ${ }^{2}$, Jianming Xu ${ }^{4}$, Jianping Xiong ${ }^{5}$, Yuxian Bai ${ }^{6}$, Zhehai Wang ${ }^{7}$, Yan Yang ${ }^{8}$, Guoping Sun ${ }^{9}$, Liwei Wang ${ }^{10}$, Leizhen Zheng ${ }^{11}$, Nong Xu ${ }^{12}$, Ying Cheng ${ }^{13}$, Weijian Guo ${ }^{14}$, Hao Yu ${ }^{15}$, Tianshu Liu ${ }^{2}$, Pagona Lagiou ${ }^{1,16^{*}}$ and Jin $\mathrm{Li}^{17^{*}}$

\section{Correction}

The original article [1] contains two errors in Table 2:

1) The data values in the rows 'Disease control rate' and 'Objective response rate' and the columns 'With adverse events' and 'Without adverse events' have mistakenly been interchanged between columns; the values ' 39 (32.77)' and ' 6 (5.04)' should be swapped with the values ' 82 (54.67)' and '11 (7.33)' respectively.

2) The value for the row 'Median progression-free survival (IQR)' for the 'HR/OR' sub-column should be 0.69 , not 0.79 .

As such, the table displayed ahead shows the correct presentation of Table 2 and should be considered instead.

\footnotetext{
Author details

'Department of Epidemiology, Harvard T. H. Chan School of Public Health, 677 Huntington Avenue, Boston, MA 02115, USA. ² Fudan University Zhongshan Hospital, Shanghai, China. ${ }^{3}$ People's Liberation Army Cancer Center, 81st Hospital of People's Liberation Army, Beijing, China. ${ }^{4}$ Academy of Military Medical Sciences, 307th Hospital of PLA, Beijing, China. ${ }^{5}$ First Affiliated Hospital of Nanchang University, Nanchang, China. ${ }^{6} \mathrm{Harbin}$ Medical University Cancer Hospital, Harbin, China. 'Thandong Cancer Hospital, Jinan, China. ${ }^{8}$ Gansu Cancer Hospital, Lanzhou, China. ${ }^{9}$ First Affiliated Hospital of Anhui Medical University, Hefei, China. ${ }^{10}$ Shanghai First People's Hospital,

* Correspondence: plagiou@hsph.harvard.edu; fudanlijin@163.com

'Department of Epidemiology, Harvard T. H. Chan School of Public Health, 677 Huntington Avenue, Boston, MA 02115, USA

${ }^{17}$ Department of Oncology, Shanghai East Hospital, Tongji University School of Medicine, No. 150 Ji Mo Road, Shanghai 200120, People's Republic of China

Full list of author information is available at the end of the article
}

Shanghai, China. ${ }^{11}$ XinHua Hospital Affiliated to Shanghai Jiaotong University, Shanghai, China. ${ }^{12}$ First Affiliated Hospital of Zhejiang University, Hangzhou, China. ${ }^{13}$ Jilin Provincial Cancer Hospital, Changchun, China. ${ }^{14}$ Fudan University Shanghai Cancer Center, Shanghai, China. ${ }^{15} \mathrm{Nanjing}$ Medical University, Nanjing, China. ${ }^{16}$ Department of Hygiene, Epidemiology and Medical Statistics, School of Medicine, National and Kapodistrian University of Athens, 75 M. Asias Street, Goudi GR, 11527 Athens, Greece. ${ }^{17}$ Department of Oncology, Shanghai East Hospital, Tongji University School of Medicine, No. 150 Ji Mo Road, Shanghai 200120, People's Republic of China.

Received: 22 November 2017 Accepted: 22 December 2017 Published online: 09 January 2018

\section{Reference}

1. Liu X, et al. Early presence of anti-angiogenesis-related adverse events as a potential biomarker of antitumor efficacy in metastatic gastric cancer patients treated with apatinib: a cohort study. J Hematol Oncol. 2017;10:153. 
Table 2 Correlation between presence of at least one anti-angiogenesis-related adverse event and antitumor efficacy of apatinib

\begin{tabular}{|c|c|c|c|c|c|c|}
\hline \multirow[t]{2}{*}{ Clinical outcomes } & \multirow{2}{*}{$\begin{array}{l}\text { With adverse events } \\
(n=150)\end{array}$} & \multirow{2}{*}{$\begin{array}{l}\text { Without adverse events } \\
(n=119)\end{array}$} & \multicolumn{2}{|c|}{ Unadjusted analysis } & \multicolumn{2}{|c|}{ Multi-adjusted analysis ${ }^{a}$} \\
\hline & & & HR/OR ${ }^{b}(95 \% \mathrm{Cl})$ & $P$ value $^{c}$ & $\mathrm{HR} / \mathrm{OR}(95 \% \mathrm{Cl})$ & $P$ value $^{d}$ \\
\hline Median overall survival (IQR), days & $169(96-255)$ & $103(58-201)$ & $0.67(0.51,0.88)$ & 0.0039 & $0.64(0.48,0.84)$ & 0.001 \\
\hline $\begin{array}{l}\text { Median progression-free survival } \\
\text { (IQR), days }\end{array}$ & $86.5(57-150)$ & $62(41-121)$ & $0.75(0.58,0.98)$ & 0.0309 & $0.69(0.53,0.91)$ & 0.007 \\
\hline Disease control rate, $n(\%)$ & $82(54.67)$ & $39(32.77)$ & $2.47(1.46,4.21)$ & $<0.001$ & $2.67(1.59,4.47)$ & $<0.001$ \\
\hline Objective response rate, $n(\%)$ & $11(7.33)$ & $6(5.04)$ & $1.49(0.49 .5 .06)$ & 0.443 & $1.42(0.50,4.01)$ & 0.505 \\
\hline
\end{tabular}

Adverse events are defined as hypertension, proteinuria, or hand and foot syndrome in the first 4 weeks of treatment

$H R$ hazard ratio, $O R$ odds ratio, IQR interquartile range

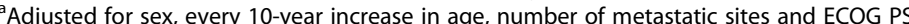

${ }^{\mathrm{b}} \mathrm{HR}$ for overall survival and progression survival; OR for disease control rate and objective response rate

${ }^{c} P$ values calculated from log-rank test for overall survival and progression survival, and chi-square test for disease control rate and objective response rate

${ }^{d} P$ values calculated from Cox regression for overall survival and progression survival, and logistic regression for disease control rate and objective response rate 